\title{
Anisotropy of Dilepton Emission from Nuclear Collisions
}

\author{
E.L. Bratkovskaya, O.V. Teryaev \\ Bogolubov Laboratory of Theoretical Physics, Joint Institute for Nuclear Research, \\ 141980 Dubna, Moscow region, RUSSIA \\ V.D. Toneev \\ Institute for Nuclear Theory, NK-12,University of Washington, \\ Seattle, WA 98195 USA \\ and \\ Bogolubov Laboratory of Theoretical Physics, Joint Institute for Nuclear Research, \\ I4I980 Dubna, Moscow region, RUSSIA
}

\section{PREPARED FOR THE U.S. DEPARTMENT OF ENERGY UNDER GRANT DE-FG06-90ER40561}

This report was prepared as an account of work sponsored by the United States Government. Neither the United States nor any agency thereof, nor any of their employees, makes any warranty, express or implied, or assumes any legal liability or responsibility for the accuracy, completeness, or usefulness of any information, apparatus, product, or process disclosed, or represents that its use would not infringe privately owned rights. Reference herein to any specific commercial product, process, or service by trade name, mark, manufacturer, or otherwise, does not necessarily constitute or imply its endorsement, recommendation, or favoring by the United States Government or any agency thereof. The views and opinions of authors expressed herein do not necessarily state or reflect those of the United States Government or any agency thereof.

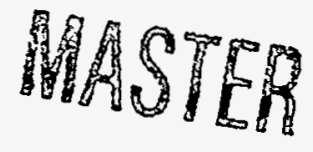

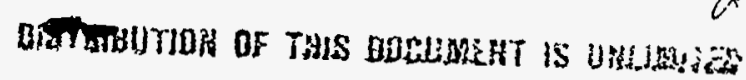




\section{DISCLAIMER}

Portions of this document may be illegible in electronic image products. Images are produced from the best available original document. 


\title{
Anisotropy of Dilepton Emission from Nuclear Collisions
}

\author{
E.L. Bratkovskaya, O.V. Teryaev \\ Bogolubov Laboratory of Theoretical Physics, Joint Institute for Nuclear Research, \\ 141980 Dubna, Moscow region, RUSSIA \\ V.D. Toneev \\ Institute for Nuclear Theory, NK-12, University of Washington \\ Seattle, WA 98195, USA \\ and \\ Bogolubov Laboratory of Theoretical Physics, Joint Institute for Nuclear Research, \\ 141980 Dubna, Moscow region, RUSSIA
}

November 7, 1994

\begin{abstract}
Attention is paid to studying the angular characteristics of $e^{+} e^{-}$pairs created in collisions with nuclear targets at intermediate and relativistic energies. Arising due to general spin and angular momentum constraints, the dilepton anisotropy seems to be quite sensitive to the contribution of different sources and may be used for disentangling these sources (or models) as well as an additional signature of a possible chiral symmetry restoration and phase transition of hadrons into the quarkgluon plasma. An anisotropy estimate for some dilepton sources is given and its relevance to the problems mentioned is discussed.
\end{abstract}


During the last years, a lot of efforts has been paid to experimental and theoretical investigations of properties of hot and dense nuclear matter. It is of great importance to search for specific processes or particular observables which are the most sensitive to nuclear equation of state, in general, or may signal us on a phase transition, in particular. In this respect, leptonic probes are quite attractive since they can provide weakly disturbed information on hot and dense nuclear matter at different stages of its evolution in heavyion collisions at both intermediate and ultrarelativistic energies. We refer to these two regimes not only for emphasizing the large scale of energy where lepton probes are effective but for noting the difference between two ways to reach an extreme nuclear state. In particular, the compession as much as three times the baryon density $\rho_{0}$ of the normal nuclear matter and not so high temperatures can be easily attained in heavy-ion collisions at the energy of about several $\mathrm{GeV} /$ nucleon. These conditions are considered as quite favorable to observe the change of hadron properties in nuclear matter related to a partial restoration of chiral symmetry. While the energy as high as $200 \mathrm{GeV} /$ nucleon is needed for observing a possible deconfinement phase transition where we have deal mainly with hot baryon-poor systems.

As to intermediate energies, some progress in this field has been made by Gale and Kapusta [1] who first pointed out that dileptons from $\pi^{+} \pi^{-}$annihilation may be very sensitive to the pion dispersion relation which strongly deviates from a free relation in dense and hot nuclear matter. As has been shown in subsequent papers $[2,3,4]$ this effect was overestimated by Gale and Kapusta. Nevertheless the main point that dilepton yield in heavy ion collisions at intermediate energies provides a unique and very attractive chance to extract information on properties of pions in dense nuclear matter, has been confirmed by the detailed kinetic calculations [5, 6, 7, 8, 9]. Even more, a very promising claim has been made $[5,6,8]$ that in heavy ion collisions at the energy of about $1 \mathrm{GeV} /$ nucleon the $e^{+} e^{-}$yield in the region of high invariant masses, $M \geq 0.5 \mathrm{GeV}$, is defined solely by the pion annihilation channel what seems to open the direct road to the experimental identification of effects of the chiral symmetry restoration. However, only dileptons from the bremsstrahlung emission and the Dalitz decay of isobars were considered here along with $\pi \pi$-annihilation. As was shown in the subsequent papers [7], hadronic sources of dileptons, i.e. $e^{+} e^{-}$pairs coming from the direct decay of produced pseudoscalar and vector mesons, are rather important even at the comparatively low colliding energy like $1 \mathrm{GeV} /$ nucleon. In particular, the direct decay of $\rho$-mesons contributes to the same region of invariant masses and as much as the pion annihilation channel [7]. In addition, the first dynamical estimate of in-medium influence on $\rho$-meson propagation through nuclear matter has demonstrated that observable effect is not so large as would be expected previously [8]. Therefore, on the one hand, this situation puts forward a very high requirement to the accuracy of future measurements of dilepton spectra. On the other hand, searching for new, more sensitive observables is necessary which would allow one to disentangle contributions of different channels.

As to ultrarelativistic energies, the situation here is somewhat similar. Actually, there is a long list of various signatures accompanying the deconfinement transition of hadrons into a quark-gluon plasma state [10]. Among the most promising signatures, one may call the strangeness enhancement as well as signs related to the measurement of lepton pairs, i.e. $J / \Psi$ suppression, $\phi / \rho$ enhancement, production of thermalized dileptons. However, 
none of these signatures is decisive and crucial since some hadronic counter-process has been found practically for every case. So, the formation of quark-gluon plasma in relativistic heavy-ion collisions can be proved only by simultaneous observation of several plasma signs. Like at intermediate energies, the search for additional plasma signatures and observables which can differentiate contributions of different processes is a very important and really intresting problem.

This paper aims to draw attention to the investigation of angular distributions of dileptons emitted from heavy-ion collisions. These observables will be estimated here for some partial channels and the definite conclusions can be drawn in a general case.

In hadron-hadron collisions, the $e^{+} e^{-}$pairs are created due to electromagnetic decay of time-like virtual photons. In turn, these virtual photons can result from the bremsstrahlung process or come from the decay of baryonic and mesonic resonances including the direct conversion of vector mesons into virtual photons in accordance with the vector dominance hypothesis. In its rest frame, the decay of an unpolarized photon gives rise to isotropy of the angular distribution for a created lepton pair since there is no preferential direction. However, the coupling of the virtual photon to hadrons may induce some dynamical spin alignment of both resonances and virtual photons. So, one can expect that the angular distribution of a lepton (say, $e^{-}$) will be anisotropic with respect to the direction of dilepton (i.e. a virtual photon) emission. This decay anisotropy defined for the given dilepton mass is carrying some information on the spin alignment of the virtual photon as well as on spins of colliding or decaying hadrons and thereby allows one to disentangle different production processes, in principle.

We propose this observable for discriminating different dilepton sources in heavy-ion collisions at intermediate energies. In the case of ultrarelativistic energies, the dilepton production from quark-antiquark annihilation in the thermalized quark-gluon is expected to be completely isotropic. So, the absence of anisotropy for enhanced dilepton emission may serve as an additional signature of the plasma formation as was noted first in ref.[11].

Now we shall exemplify our general consideration of symmetry arguments by the calculation results for specific channels. This consideration is based in part on our previous paper [12], so we shall not go here into details of this microscopic calculations. To characterize the decay anisotropy, let choose the polar $\theta$ and azimuthal $\varphi$ angles of the momentum $\vec{l}_{-}$of a created electron with respect to the momentum $\vec{q}$ of a virtual photon in the rest frame of this virtual photon, i.e. $\vec{l}_{-}+\vec{l}_{+}=0$. For comparing the shape of the angular distribution for different channels, it is convenient to represent the differential cross section for dilepton production in the following form:

$$
S(M, \theta) \equiv \frac{d \sigma}{d M^{2} d \cos \theta}=A\left(1+B \cos ^{2} \theta\right),
$$

where $M$ is the invariant mass of a lepton pair $\left(M^{2}=q_{0}^{2}-\vec{q}^{2}\right)$. So, the anisotropy coefficient $B$ can be found as

$$
B=\frac{S\left(M, \theta=0^{\circ}\right)}{S\left(M, \theta=90^{\circ}\right)}-1 .
$$

Being defined by the spin structure of interaction process, the coefficient $B$ can be, in a general case, a function of $M$ and masses of particles involved into the reaction. 
The Dalitz decay of a pseudoscalar meson, say $\eta$, is one of the simplest cases since the zero spin of the meson separates the spin indices of the initial and final states allowing one to factorize the production $(N N \rightarrow \eta X)$ and decay $\left(\eta \rightarrow \gamma e^{+} e^{-}\right)$amplitudes. In particular, the angular distribution can be written as follows:

$$
\frac{d \sigma^{\eta}}{d M^{2} d \Omega}=\sigma^{\eta}(s) \frac{1}{\Gamma_{t o t}^{\eta}} \frac{d \Gamma^{\eta \rightarrow e^{+} e^{-} \gamma}}{d M^{2} d \Omega}
$$

where $\sigma^{\eta}(s)$ is the $\eta$-production cross section depending on the total energy squared $s$ of colliding nucleons, $\Gamma_{\text {tot }}^{\eta}$ is the total decay width. Here the $\eta$-decay probability has the form:

$$
\frac{d \Gamma^{\eta \rightarrow e^{+} e^{-} \gamma}}{d M^{2} d \Omega}=\frac{2 \alpha}{3 \pi^{2}} \frac{B r^{\eta} \Gamma_{t o t}^{\eta}}{m_{\eta}^{4}}\left|T^{\eta \rightarrow e^{+} e^{-} \gamma}\right|^{2}\left(\frac{\lambda\left(m_{\eta}^{2}, M^{2}, 0\right)}{\lambda\left(m_{\eta}^{2}, 0,0\right)}\right)^{1 / 2}
$$

with the triangle kinematical function $\lambda(x, y, z)=x^{2}+y^{2}+z^{2}-2(x y+y z+x z)$ and the branching ratio $B r^{\eta}$ for the $\eta \rightarrow \gamma \gamma$ decay [13]. The transition matrix element is given by the following relation:

$$
\left|T^{\eta \rightarrow e^{+} e^{-} \gamma}\right|^{2} \sim \frac{1}{M^{4}}\left(\epsilon_{\mu \alpha \beta \gamma} q_{\beta} q_{1_{\gamma}} \epsilon_{\nu \alpha \rho \sigma} q_{\rho} q_{1_{\sigma}}\right) \cdot L_{\mu \nu}
$$

where $q, q_{1}$ are the four momenta of a virtual and real photon, respectively. Here, the lepton tensor $L_{\mu \nu}$ is defined as

$$
L_{\mu \nu}=\operatorname{Tr} \hat{l}_{-} \gamma_{\mu} \hat{l}_{+} \gamma_{\nu}
$$

and $l_{-}, l_{+}$are the four momenta of leptons. As follows from Eq.(5), the $\eta$-decay probability does not depend on the azimuthal angle $\varphi$. For the polar angle, this distribution is reduced to (2) with $B=1$, so

$$
\frac{d \sigma^{\eta}}{d M^{2} d \cos \theta} \sim 1+\cos ^{2} \theta
$$

One should notice that the same answer results really from the decay of any pseudoscalar meson into $\gamma$ and vector meson, $P \rightarrow V \gamma$, with the subsequent $\gamma$ conversion into a $e^{+} e^{-}$ pair. It is valid, in particular, for the $\pi^{0} \rightarrow e^{+} e^{-} \gamma$ decay which dominates at small invariant masses $M \leq 0.14 \mathrm{GeV}$.

For the Dalitz decay of a delta resonance, $\Delta \rightarrow e^{+} e^{-} N$, formed via the $N N \rightarrow$ $\Delta N$ process, the dilepton differential cross section cannot be reduced to the form of eq.(3) due to $3 / 2$ spin of the delta. Even more, yet early experiments [14] have shown that, for example, a $\Delta^{++}$isobar coming from $p p$ collisions at $2.8 \mathrm{GeV} / \mathrm{c}$ is produced in some aligned state. Nevertheless, if this alignment effect is neglected for a time ${ }^{1}$, we get in this very crude approximation

$$
\frac{d \sigma^{\Delta}}{d M^{2} d \Omega}=\int d M_{\Delta} \sigma_{\Delta}\left(s, M_{\Delta}\right) D\left(M_{\Delta}\right) \frac{1}{\Gamma_{\text {tot }}^{\Delta}} \frac{d \Gamma^{\Delta \rightarrow e^{+} e^{-N}}}{d M^{2} d \Omega}
$$

\footnotetext{
${ }^{1}$ Full calculations are in progress now.
} 
where $\sigma_{\Delta}\left(s, M_{\Delta}\right)$ is the cross section of delta production with mass $M_{\Delta}$ [12]. In contrast with the $\eta$ Dalitz decay having a very narrow decay width $\left(\Gamma_{\text {tot }}^{\eta}=1.19 \mathrm{KeV}\right)$, the mass distribution of a virtual delta $D\left(M_{\Delta}\right)$ is additionally taken into account in (8). The function $D\left(M_{\Delta}\right)$ was taken from ref.[12] and $\Gamma_{\text {tot }}^{\Delta}$ is the total decay width. The $\Delta$-decay probability has the form:

$$
\frac{d \Gamma^{\Delta \rightarrow e^{+} e^{-} N}}{d M^{2} d \Omega}=\frac{\alpha^{2}}{64 \pi^{2} M^{4}}\left|T^{\Delta \rightarrow e^{+} e^{-N}}\right|^{2} \frac{\lambda\left(M_{\Delta}^{2}, m_{N}^{2}, M^{2}\right)}{M_{\Delta}^{3}} .
$$

For the amplitude squared we get

$$
\left|T^{\Delta \rightarrow e^{+} e^{-N}}\right|^{2} \sim \frac{1}{M^{4}} \operatorname{Tr}\left(\hat{P}_{\Delta}+M_{\Delta}\right) \Lambda_{\alpha \beta} \Gamma_{\beta_{\mu}}\left(\hat{p}_{a}^{\prime}+m_{N}\right) \Gamma_{\alpha \nu} \cdot L_{\mu \nu},
$$

where $p_{a}$ and $p_{a}^{\prime}$ are the four momenta of initial and outgoing nucleons, respectively, $P_{\Delta}$ is the four momentum of the virtual delta isobar and $\left(P_{\Delta}+M_{\Delta}\right) \Lambda_{\alpha \beta}$ is the projector on the state with spin $3 / 2$ (for example, see ref. [15]):

$$
\Lambda_{\alpha \beta}=g_{\alpha \beta}-\frac{2}{3 M_{\Delta}^{2}} P_{\Delta_{\alpha}} P_{\Delta \beta}-\frac{1}{3} \gamma_{\alpha} \gamma_{\beta}+\frac{1}{3 M_{\Delta}}\left(P_{\Delta_{\alpha}} \gamma_{\beta}-P_{\Delta_{\beta}} \gamma_{\alpha}\right)
$$

The vertex function $\Gamma_{\beta \mu}$ is defined similarly to ref. [16]:

$$
\begin{aligned}
& \Gamma_{\beta \mu}=\frac{-3 \varepsilon_{\beta \mu}\left(M_{\Delta}+m_{N}\right)}{2 m_{N}\left[\left(M_{\Delta}+m_{N}\right)^{2}-M^{2}\right]}, \quad \varepsilon_{\beta \mu}=-M_{\Delta} \Gamma_{\beta \mu}^{1}+\Gamma_{\beta_{\mu}}^{2}+\frac{1}{2} \Gamma_{\beta \mu}^{3}, \\
& \Gamma_{\beta \mu}^{1}=\left(q_{\beta} \gamma_{\mu}-g_{\beta \mu} \hat{q}\right) \gamma_{5}, \\
& \Gamma_{\beta \mu}^{2}=\frac{1}{2}\left(q_{\beta}\left(p_{a}^{\prime}+P_{\Delta}\right)_{\mu}-g_{\beta \mu} q \cdot\left(p_{a}^{\prime}+P_{\Delta}\right)\right) \gamma_{5}, \\
& \Gamma_{\beta \mu}^{3}=\left(q_{\beta} q_{\mu}-g_{\beta \mu} q^{2}\right) \gamma_{5} .
\end{aligned}
$$

After calculations we arrived again at the distribution (7). Note that this unexpected answer results from the dominance of the magnetic form factor $\Gamma_{\beta_{\mu}}^{1}$ and the crude assumption (8) which is rather succesful, however, in describing the invariant mass distribution of dileptons. By this example we would like to stress a high sensitivity of anisotropy to the spin structure of the transition matrix element rather then to state that the angular distribution for the Dalitz delta decay source follows the form (7).

Dilepton production in bremsstrahlung processes can be estimated in the soft photon approximation. In the case of nucleon-nucleon collisions, $N N \rightarrow e^{+} e^{-} N N$, we have for the cross section [12]:

$$
\frac{d \sigma^{\gamma}}{d M^{2} d \Omega} \sim \int \frac{d V}{j_{\text {in }}}\left|T^{\gamma}\right|^{2} \cdot\left|T^{\mathrm{e}}\right|^{2}
$$

where $T^{\text {el }}$ is the amplitude of elastic $N N \rightarrow N N$ scattering and the electomagnetic matrix element is defined as

$$
\left|T^{\gamma}\right|^{2}=J_{\mu} J_{\nu} \cdot L^{\mu \nu}
$$




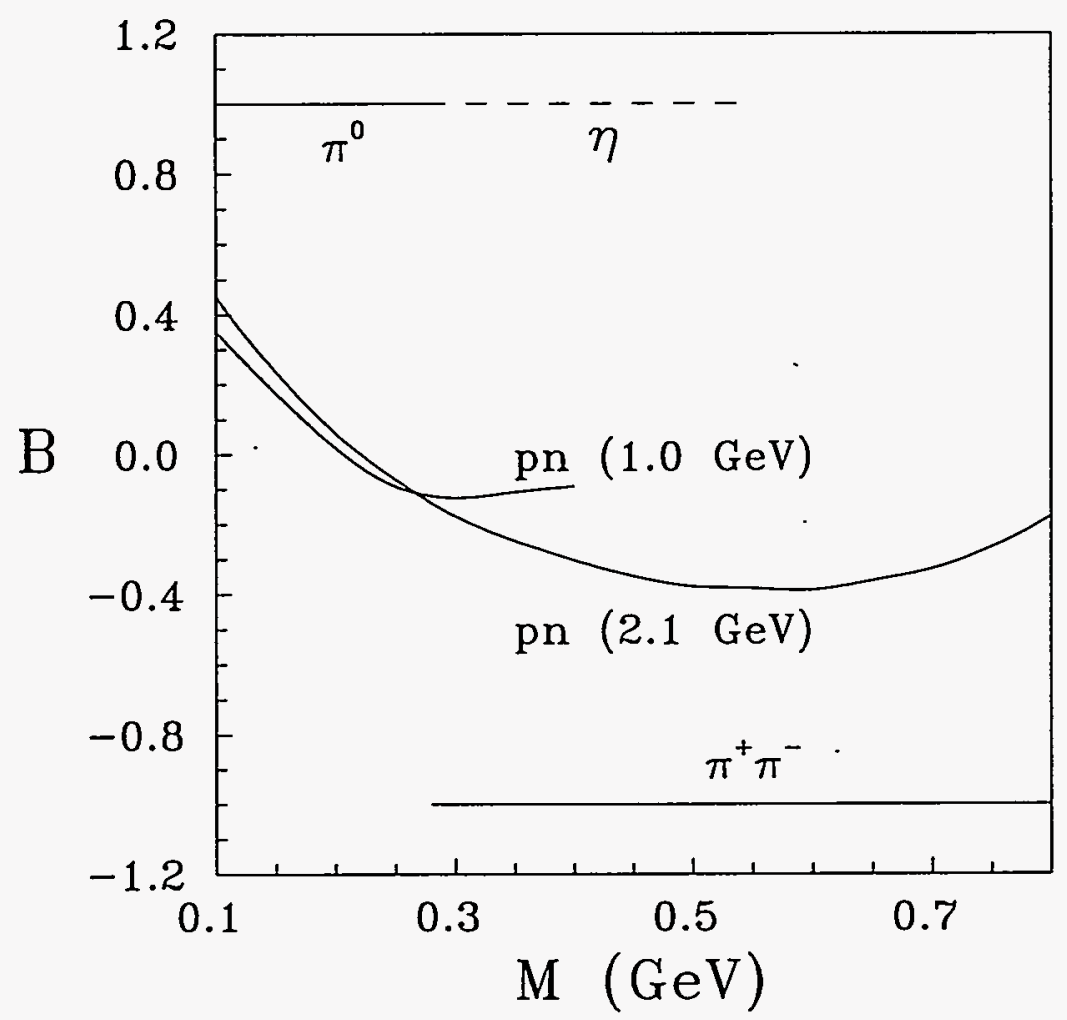

Figure 1: $M$ dependence of the decay anisotropy coefficient for different elementary dilepton sources: the Dalitz decay of $\eta$ and $\pi^{0}$ mesons, $\pi^{+} \pi^{-}$-annihilation and $p n$ bremsstrahlung (at two energies).

In the soft photon approximation, the hadron current $J_{\mu}$ for the $p n$ bremsstrahlung is given by the simple relation

$$
J_{p n}^{\mu}=-\frac{p_{a}^{\mu}}{\left(p_{a} \cdot q\right)}+\frac{p_{a}^{\prime \mu}}{\left(p_{a}^{\prime} \cdot q\right)} .
$$

The calculated dilepton anisotropy coefficient $B$ is shown in Fig.1. One can see that $B$ is the $M$ dependent quantity and changes the sign above $M \sim 0.2 \mathrm{GeV}$. The distribution over the azimuthal angle $\varphi$ is not isotropic what introduces axial symmetry breaking. So, the coefficient $B$ is getting now the function of initial energy, invariant mass and azimuthal angle $\varphi, B=f(s, M, \varphi)$.

Let us proceed to the pion annihilation process, $\pi \pi \rightarrow e^{+} e^{-}$, which is expected to be an important source of the dileptons in the hot nuclear matter [1]. Due to the most simple kinematics it is instructive to present a derivation of the angular distribution in some details. As a result, a physical origin of the anisotropy will become more clear.

The differential cross section is just proportional to the pion form factor squared times the lepton trace $L_{\mu \nu}$ (we drop for a time the kinematical factors, which are canceled while the dimensionless anisotropy factors are calculated):

$$
\frac{d \sigma}{d t} \sim-\Delta p^{\mu} \Delta p^{\nu}\left|F_{\pi}\left(q^{2}\right)\right|^{2} L_{\mu \nu}, \quad s=M^{2} .
$$


Here again $q=p_{a}+p_{b}=l_{-}+l_{+}$is the virtual photon momentum, $t=\left(p_{a}-l_{-}\right)^{2}$, $\Delta p=p_{a}-p_{b}$ is the relative momentum of the colliding pions, which have only the space component in their c.m. frame. It is convenient to use the similar relative momentum $\Delta l=l_{-}-l_{+}$for the leptons as well:

$$
\frac{1}{4} L_{\mu \nu}=q^{2} g_{\mu \nu}-q^{\mu} q^{\nu}+\Delta l^{\mu} \Delta l^{\nu}
$$

Note the obvious relation:

$$
\Delta p \cdot q=\Delta l \cdot q=0
$$

If the lepton mass is neglected, the following relation is also valid:

$$
(\Delta l)^{2}=-q^{2}
$$

Making use of these relations one can easily transform (16):

$$
\frac{d \sigma}{d t} \sim q^{2}(\Delta p)^{2}-(\Delta p \cdot \Delta l)^{2}
$$

Passing now to the center-of-mass frame, which coincides with the rest frame of a virtual photon $\vec{q}=0$, we arrive at the following angular distribution

$$
\frac{d \sigma}{d \cos \theta} \sim 1-\cos ^{2} \theta
$$

where $\theta$ is the angle between the directions of $\Delta p$ and $\Delta l$, both possessing the space components only in this frame. The anisotropy coefficient $B=-1$ is also plotted in Fig.1. As is seen, the $B$ values are ranging from +1 to -1 and depend on both invariant mass $M$ and the process considered. The observed $B$ will be a convolution of these values with $d \sigma / d M$ for every source. However, even without detailed calculations one can predict that, in particular, for heavy ion collisions at the energy of $1 \mathrm{GeV} /$ nucleon the $B$ coefficient should change the sign around $M \sim(0.4-0.5) \mathrm{GeV}$ getting negative for higher invariant masses $M$ due to the contribution of $\pi \pi$ annihilation. But in contrast with other sources, the asymmetry coefficient $B$ for $\pi \pi$ annihilation in nuclear collisions depends on an effective spectrum of colliding pions since the center-of-mass and the virtual photon systems coincide each with other, as has been noted above. So, the B values in the $\rho$-meson region of $M$ will be different for the $C a+C a$ and $A u+A u$ collisions and if the full isotropy, $B \approx 0$, is observed, it would be a signal on thermalization of the pionic component. Therefore, we expect that the measured $M$-dependent shape of the decay angular distributions will be more sensitive to the contribution of different dilepton sources as compared to, for example, slowly varying transverse momentum distributions, $d \sigma / d M^{2} d p_{t}^{2}$, measured recently for dileptons [17]. We hope that this new observable will allow one to discriminate different models $[2,5,6,7]$ which fit almost equally well (within rather large experimental error bars) the available data on dilepton production in heavy-ion collisions at intermediate energies but predict different contributions of dilepton sources. Anisotropy analysis can help also in solving the puzzling ratio of $d \sigma / d M$ for dileptons coming from $p D$ and $p p$ collisions [18]. 
The lepton angular anisotropy may be considered as an analyzer of the tensor polarization (alignment) of the virtual photon. The cross section (16) can be rewritten then like

$$
\frac{d \sigma}{d t} \sim \rho^{\mu \nu} L_{\mu \nu}
$$

where

$$
\rho^{\mu \nu}=-\Delta p^{\mu} \Delta p^{\nu}\left|F_{\pi}\left(q^{2}\right)\right|^{2}
$$

is the virtual photon spin density matrix. The vector (circular) polarization is not contribute, because it corresponds to the antisymmetric part of the spin density matrix.

Turning to high energies, let us consider the Drell-Yan process, when quarks annihilate into a lepton pair, $q \bar{q} \rightarrow e^{+} e^{-}$. The result differs significantly from the pion annihilation case. While the lepton part $L_{\mu \nu}$ is completely the same, the photon density matrix will be proportional to the quark trace which, in its turn, is analogous to the lepton one

$$
\rho_{\mu \nu}=q^{2} g_{\mu \nu}-q^{\mu} q^{\nu}+\Delta p^{\mu} \Delta p^{\nu}
$$

As a result, the familiar expression is reproduced immediately:

$$
\frac{d \sigma}{d \cos \theta} \sim 1+\cos ^{2} \theta
$$

Such a difference between quark and pion annihilation is very simple to understand using the helicity and angular momentum conservation. Let quark and antiquark have opposite momenta along $z$ direction. The massless quark and antiquark can annihilate only in the states with the opposite helicities. Therefore, their spins are aligned in the same direction and by angular momentum conservation (the orbital angular momentum $z$ component is zero) the $z$ component of them is \pm 1 . This corresponds to the spin density matrix element $\rho_{00}^{z}=0$. For the annihilation of the spinless pions the $z$ component of the virtual photon spin is zero $\left(\rho_{00}^{z}=1\right)$. Note that the zero spin in the initial state does not lead to the vanishing of all the components of photon spin because of the orbital angular momentum contribution.

The different shape of the lepton angular distributions in these two cases is due to the unique spin structure of the amplitude of virtual photon production defining its spin density matrix. This is also in the case of the Dalitz decay of the pseudoscalar meson, where distribution (21) is also valid.

In general, the dependence on the azimuthal angle $\varphi$ can be manifested as well:

$$
\frac{d \sigma}{d \cos \theta d \varphi}=N\left(1+a \cos ^{2} \theta+b \sin 2 \theta \cos \varphi+c \sin ^{2} \theta \cos 2 \varphi\right),
$$

where $N, a, b, c$ are angle-independent. Note that nonzero $b$ and $c$ require the violation of the axial symmetry with respect to the $z$ axis. This is the case, if some other particle(s) is produced in addition to dilepton and scattering plane of the virtual photon production is defined. In this case one should take care to don't miss the decay lepton angles (which 
should be measured in the dilepton c.m. frame) and the virtual photon production angles measured in the c.m. frame of colliding particles. These two frames are not more coincide. The decay lepton angles directly measure the virtual photon spin density matrix, while the photon angles characterize the production process. The last angles are also of interest for disentangling dilepton sources. However, since in heavy-ion reactions we have deal with the superposition of many hadronic collisions at different energies, it is preferable to represent distributions over the dilepton production angle in the equal-velocity system of colliding nuclei.

In concluding, let us discuss the influence of surrounding nuclear matter on the anisotropy characteristics. This influence is reduced to the effects of two kinds. First one may be related to a possible in-medium modification of the form factors, vertices and decay width of hadrons but it does not change really the spin structure of interaction. So, the hadronic sources will have the same anisotropy but their relative contributions will be changed what opens the way to get some feeling as to the strength of in-medium effects. The second modification is due to a possible appearence of some new phase (source) characterized by a four-velocity $v^{\mu}[19,20]$. This results in the appearance of additional form factors spoiling some definite predictions. For the case of the pion annihilation, eq.(16) can be modified as

$$
\begin{aligned}
\frac{d \sigma}{d t} & \sim-\left(\Delta p^{\mu} F_{\pi}\left(q^{2}, v q, v \Delta p\right)+\left(v^{\mu}-q^{\mu} \frac{v q}{q^{2}}\right) F_{\pi}^{\prime}\left(q^{2}, v q, v \Delta p\right)\right) \times \\
& \times\left(\Delta p^{\nu} F_{\pi}^{*}\left(q^{2}, v q, \Delta p q\right)+\left(v^{\nu}-q^{\nu} \frac{v q}{q^{2}}\right) F_{\pi}^{\prime *}\left(q^{2}, v q, v \Delta p\right)\right) L_{\mu \nu} .
\end{aligned}
$$

So, the cross section takes the form:

$$
\begin{aligned}
\frac{d \sigma}{d t} & \sim\left|F_{\pi}\right|^{2}\left(q^{2}-\frac{(\Delta p \Delta l)^{2}}{(\Delta l)^{2}}\right)-\left|F_{\pi}^{\prime}\right|^{2}\left(1-\frac{(v q)^{2}}{q^{2}}+\frac{(v \Delta l)^{2}}{(\Delta l)^{2}}\right)- \\
& -2 \operatorname{Re}\left(F_{\pi}^{\prime} F_{\pi}^{*}\right)\left(v \Delta p+\frac{(v \Delta l)(\Delta p \Delta l)}{(\Delta l)^{2}}\right) .
\end{aligned}
$$

Eliminating the dependence on lepton angles, one has:

$$
\frac{d \sigma}{d \cos \theta} \sim 1+A \cos ^{2} \theta+B \cos ^{2} \theta^{\prime}+C \cos \theta \cos \theta^{\prime},
$$

where $\theta^{\prime}$ is the angle between the directions of $\Delta l$ and $v$. Unfortunately, one cannot say much about this new form factor $\left(F_{\pi}^{\prime}\right.$. However, at intermediate energies where the pion annihilation channel is considered as the most promising probe of chiral restoration symmtry, the medium velocity should be small (at least in average) in the lepton c.m. frame, leading to values of $B, C$ much smaller than $A$.

Somewhat more definite conclusions can be done for $q \bar{q}$ annihilation in the quark-gluon plasma. At finite temperature and density, the dilepton production can be described by the modified photon propagator which have the following invariant-tensor decomposition [20]:

$$
W^{\mu \nu}=W_{1}\left(q^{2}, v q\right)\left(g^{\mu \nu}-\frac{q^{\mu} q^{\nu}}{q^{2}}\right)+W_{2}\left(q^{2}, v q\right)\left(v^{\mu}-q^{\mu} \frac{v q}{q^{2}}\right)\left(v^{\nu}-q^{\nu} \frac{v q}{q^{2}}\right)
$$


where $W_{1}$ and $W_{2}$ are entirely analogous to the structure functions of deep-inelastic lepton scattering from nuclei (except that here the virtual photon has a time-like momentum while in deep inelastic scattering the momentum is space-like). Contracting it with the lepton trace we have

$$
\frac{d \sigma}{d t} \sim 2 W_{1}+W_{2}\left(1-\frac{(v q)^{2}}{q^{2}}+\frac{\left.(v \Delta l)^{2}\right)}{(\Delta l)^{2}}\right)
$$

or in the lepton c.m. frame

$$
\frac{d \sigma}{d \cos \theta} \sim 1+\frac{|\vec{v}|^{2}}{2 \frac{W_{1}}{W_{2}}+1-\frac{(v q)^{2}}{q^{2}}} \cos ^{2} \theta,
$$

The angle is now measured with respect to the direction of the plasma velocity $\vec{v}$. This formula justifies the earlier suggestion [11] about the $\cos ^{2} \theta$ distribution of dileptons produced from quark-gluon plasma. One can easily see that distribution becomes isotropic if plasma is at rest [11] and also for high plasma temperature, because $W_{2}$ turns out to be zero [20]. The azimuthal angle dependence (22) is of special interest, because it can never been attributed to quark-gluon plasma. Its vanishing may be considered as a new signal of the plasma formation.

One should note that the proposed dilepton observables can be estimated experimentally by new processing of the available DLS data [17, 18,21] but certainly the large acceptance and high statistics measurements are needed. In this respect, the experimental setups of new generation, HADES at Darmstadt and CERES at CERN, seem to be very promising.

We are indebted to A.M. Baldin, W. Cassing, V. Emel'yanov, V. Koch, U. Mosel, S.B. Nurushev, J. Soffer, A.I. Titov for stimulating discussions and comments. We are thankfull to S.S. Shimanskiy who paid our attention to CERES experimental facilities and pushed us to studing this problem. One of authors (V.T.) thanks the Institute for Nuclear Theory at the University of Washington for its hospitality and the Department of Energy for partial support during the completion of this work. This research was possible in part due to Grant $N_{-}^{0}$ MP8000 from International Science Foundation. 


\section{References}

[1] C. Gale and J. Kapusta. Phys. Rev. C35 (1985) 2107; Phys. Rev. C40 (1988) 745.

[2] L.H. Xia, C.M. Ko, L. Xiong and J.Q. Wu, Nucl. Phys. A485 (1988) 721.

[3] C.L. Korpa and S. Pratt, Phys. Rev. Lett. 64 (1990) 1502; C.L. Korpa at al., Phys. Lett. B246 (1990) 333.

[4] M. Herrmann, B. Friman and W. Nörenberg, Nucl. Phys. A545 (1992) 267c.

[5] L. Xiong, Z.G. Wu, C.M. Ko and J.Q. Wu, Nucl. Phys. A512 (1990) 772.

[6] Gy. Wolf, G. Batko, W. Cassing, et al., Nucl. Phys. A517 (1990) 615.

[7] V.D. Toneev, K.K. Gudima and A.T. Titov, Preprint GSI-92-05, Darmstadt, 1992; Sov. Jour. of Nucl. Phys. 55 (1992) 1715; K.K. Gudima, A.I. Titov and V.D. Toneev, Phys. Lett. B 287 (1992) 302.

[8] Gy. Wolf, W. Cassing, W. Ehehalt and U. Mosel, Progr. Part. Nucl. Phys. 30 (1993) 273.

[9] L.A. Winkelmann, H.Sorge, H. Stöcker and W.Greiner, Phys. Lett. B298 (1993) 22.

[10] Quark Matter '93. Proceedings of the Tenth International Conference on UltraRelativistic Nucleus-Nucleus Collisions, Borlänge, Sweden, June 20-24, 1993; Nucl. Phys. A566 (1994).

[11] P. Hoyer, Phys. Lett. B187 (1987) 162.

[12] B. Kämpfer, A. I. Titov and E. L. Bratkovskaya, Phys. Lett. B301 (1993) 123.

[13] Particle Data Group. Review of Particle Properties. Phys. Lett. B239 (1990).

[14] T.C. Bacon et al, Phys. Rev. 162 (1967) 1320.

[15] T. Ericson and W. Weise, Pions and Nuclei, Clarendon Press, Oxford, 1988.

[16] H. F. Jones and M. D. Scadron, Ann. Phys. 81 (1973) 1.

[17] H.Z. Huang, S. Beedoe, M. Bougteb at al., Phys. Rev. C49 (1994) 314.

[18] W.K. Wilson et al., Phys. Lett. B316 (1993) 245.

[19] A.M. Baldin, Fortsch. der Physik 38 (1990) 261.

[20] L.D. McLerran and T. Toimela, Phys. Rev. D31 (1985) 545.

[21] G. Roche, J. Bystricky, J. Carrol at al., Phys. Lett. B226 (1989) 228. 